تأثير زمن الترسيب ودرجة الحرارة على الخصائص التركيبية والطبوغرافية والبصرية لغثاء (ZnS) النانوي

المحضر بأستخدام تقنية الترسيب بالحمام الكيميائي (CBD)

\author{
عبد المجيد عيادة إبراهيم \\ تحسين علي أسود نوفل يوسف جميل \\ قسم الفيزياء/ كلية التربية للعلوم الصرفة/ جامعة تكريت

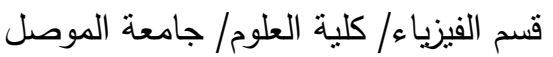

(أستلم 2018/12/16 ؛ قُبل 2019/ 15 /

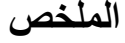

تضمن البحث دراسة تأثير بعض عوامل التحضير (زمن الترسيب ودرجة الحرارة) على الخصائص التركيبية والطبوغرافية

والبصرية لغشاء كبريتيد الخارصين (ZnS) النانوي. الأغشية النانوية حضرت بأستخدام طريقة ترسيب الحمام الكيميائي. وقد

تضمنت دراسة الخواص التركيبية: صوراً لحيود الأشعة السينية (XRD)، كما تضمنت الخواص الطبوغرافية: صوراً للمجهر

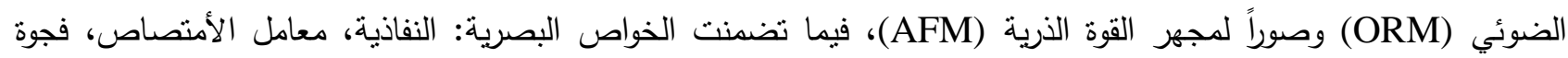

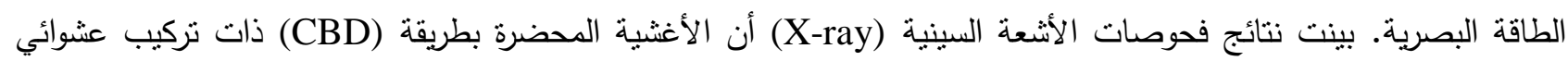

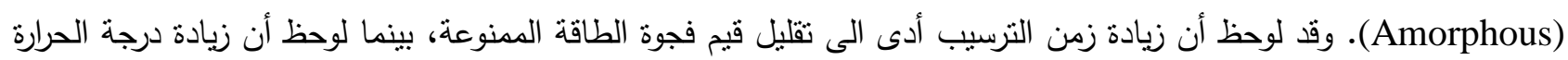
أدى الى زيادة قيم فجوة الطاقة الممنوعة.

الكلمات الدالة: الترسيب بالحمام الكيميائي، ZnS، أغثية نانوية، زمن الترسيب، درجة الحرارة.

\section{Effect of Deposition Time and Temperature on Structural, Topographic and Optical Properties of Nano (ZnS) Film Prepared by CBD Technique}

\author{
Tahseen A. Aswad \\ Nawfal Y. Jamil \\ Department of Physics/ College of Science/ University of Mosul \\ Abdul-Majeed E. Ibrahim \\ Department of Physics/ College of Education for Pure Science/ University of Tikrit
}

\begin{abstract}
The research includes the study of the effect of some preparing factors (time of deposition and temperature) on structural, topographic and optical properties of zinc sulfide ( $\mathrm{ZnS})$ nanoscale film. Nanofilms prepared by using a chemical bath deposition .The compositional properties included: pictures of X-ray diffraction (XRD), also included the topographic characteristics: pictures of the optical microscope (ORM) and pictures of the atomic force microscope (AFM), while the included optical properties: transmittance, absorption coefficient, the optical energy gap. The results of $\mathrm{X}$-ray examinations (X-ray) to prepare films by (CBD) method have amorphous structure. It has been observed that increasing the time of deposition led to decrease values of the forbidden energy gap, while it was observed that the increase in temperature led to a increase in the values of the forbidden energy gap .
\end{abstract}

Keywords: chemical bath deposition, ZnS, Nano films, Deposition Time, Temperature. 


\section{المقدمة}

لقد اتجهت الصناعة في العقود الأخيرة من القرن العشرين نحو تطوير منتجاتها من حيث تقليل كلف إنتاجها وتحسين كفاءتها وتسهيل تصنيعها وتقليص حجومها (الجمال وال فليح، 2006). وبسبب التكلفة المتزايدة لعمليات انتاج وتصنيع الاجهزة الاككترونية كانت ولا تزال هناك محاولات بهدف استبدال المواد شبه الموصلة الكتلية (Bulk Materials) بطبقات من الأغشية. ولأهمية أغثية أثنباه الموصلات، عمل الباحثون على اكتشاف طرق ترسيب جديدة وتطوير الطرق العملية المتعارف عليها، ولقد تفاوتت الخصائص الفيزيائية للأغشية تبعا لطريقة الترسيب (Nadeem et al., 2005).

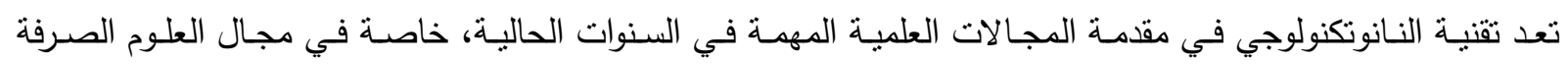

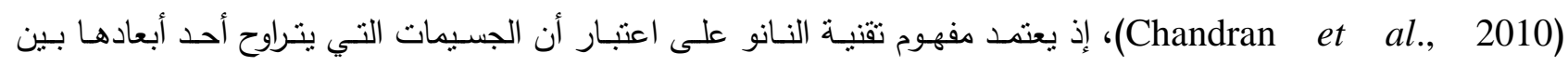

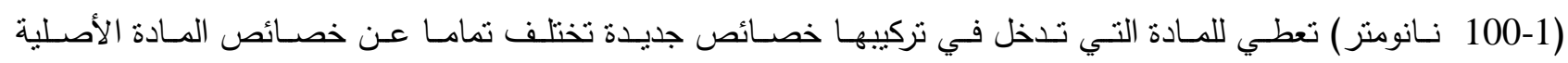

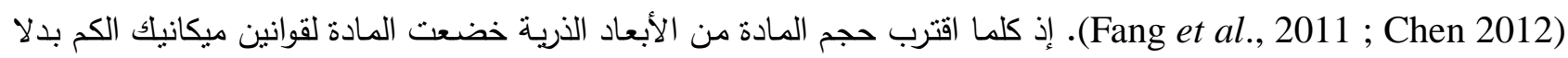
مـ قوانين الفيزيـاء الكلاسيكية. يمكن تحضير التراكيب النانويـة (Nanostructures) بأثكال مختلفـة مثنل الأقراص النانويـة

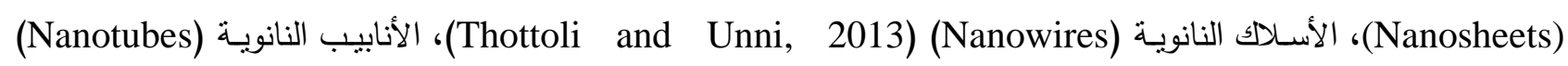
(Wu et al., 2003) النانوية (Guixiang, 2009; Ma et al., 2004; Üzar and Arikan, 2010) (Nanobelts) والكيبلات النانوية .(Suresh, 2013) (Nanocables)

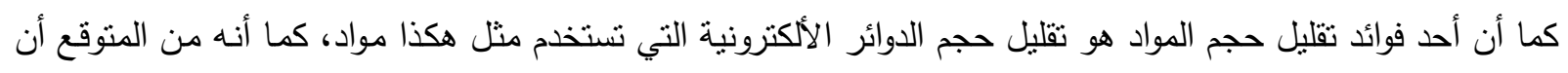

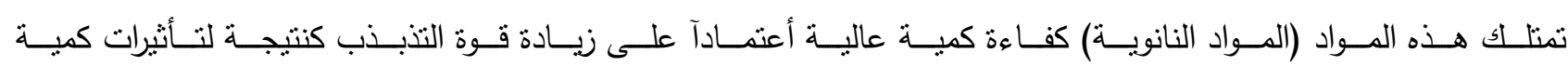

.(Gayou1 et al., 2010)

\section{الجاتب العملي والحسابات}

تم تحضير أغشية كبريتيد الخارصبن (ZnS) بطريقة الترسيب في حمام كيميائي على أرضيات (substrates) زجاجية

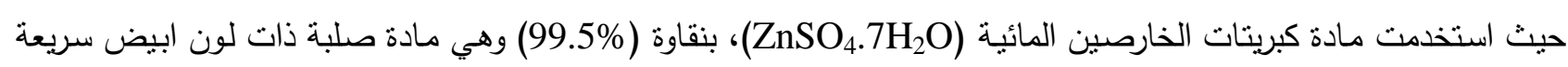
الذوبان في الماء، وزنها الجزيئي (CS(

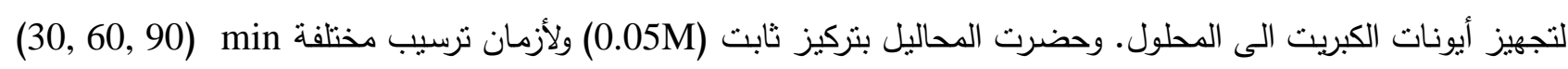
بثبوت درجة الحرارة عند (70 (70)، كما نم تغيير درجات الحرارة عند (0.05)، وذللك بإذابة الوزن المطلوب من المادة في (20 ml) من الماء المقطر اذابة تدريجية باستخدام خلاط مغناطيسي في درجة حرارة الغرفة لمدة (Magnetic Stirrer)

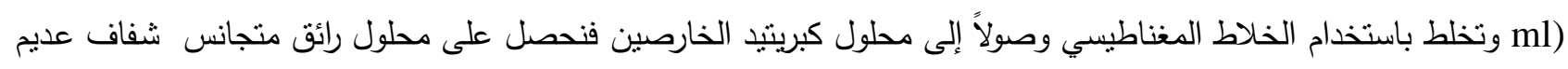

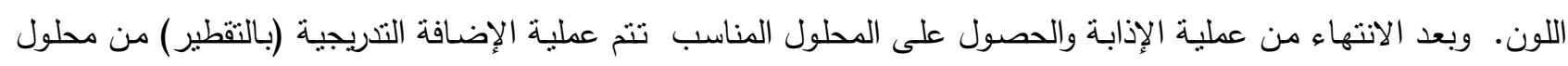
هيدروكسيد الامونيوم (NH4) كعامل مساعد للتفاعل بتركيز (30\%) ثم يتغير لون المحلول الثفاف الى اللون المعتم لفترة قصيرة، بعدها ونتيجة الأستمرار بالخلط بأستخدام الخلاط المغناطيسي يرجع المحلول الى محلى لـول رائق متجانس شفاف عديم اللون

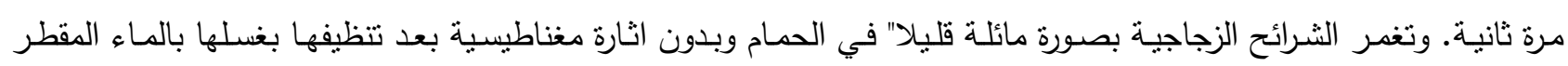

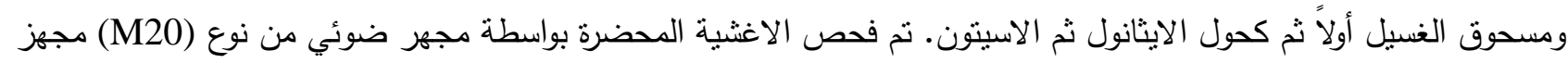
من شركة Phywe الألمانية للتعرف على طبيعة سطح الغشاء من حيث خلوه من الفراغات والثقوب الابرية، وقد تبين من نتيجة

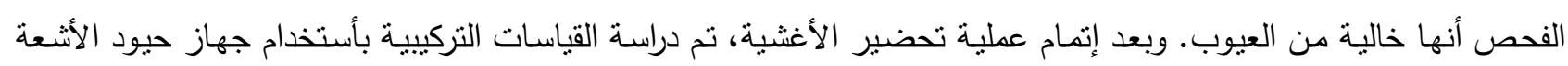
السينية نوع (Siemens X-ray diffractometer)، كما تم دراسة القياسات الطبوغرافية للسطح بأستخدام مجهر القوة الذرية دراسية 


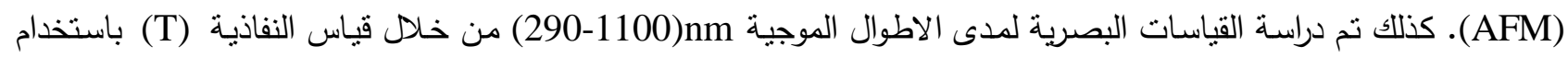

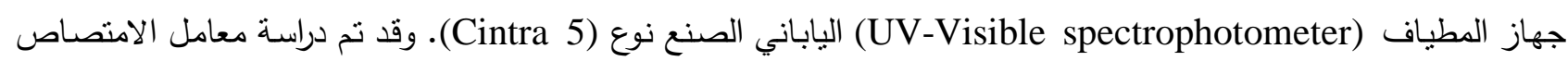
ومعامل الخمود وفجوة الطاقة من العلاقات الاتية حسب التعاقب:

$\alpha=2.3026 \mathrm{~A} / \mathrm{t}$

$\mathrm{k}_{0}=\alpha \lambda / 4 \pi$

$\alpha h v=\mathrm{k}\left(\mathrm{hv}-\mathrm{E}_{\mathrm{g}}\right)^{1 / 2}$

حيث أن:

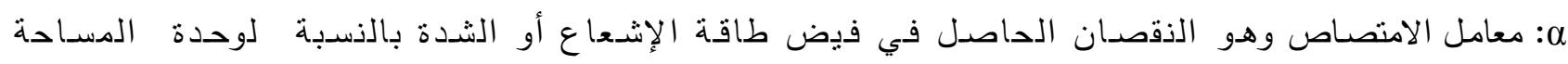
باتجاه انتشار الموجة داخل الوسط.

A الامتصاصية وهي النسبة بين الثدة الممتصة (IA) والثدة الواصلة للنموذج (Io) (Stenzel, 2005).

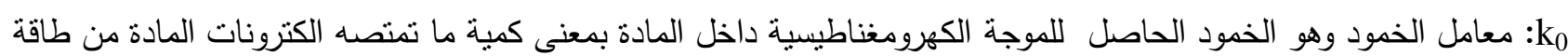

الفوتونات الساقطة (Eya et al., 2005).

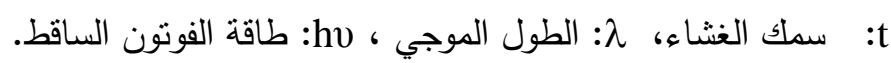
Eg

\section{النتائج والمناقشة}

سمك الغثاء

نم حساب سمك الأغشية المحضرة بأستخدام الطريقة الوزنية حيث أستعمل ميزان حساس لذلك، وقد لوحظ بأن سمك الغشاء يقل مع زيادة درجة الحرارة ويعزى سبب ذلك الى كون الحرارة عادةً تساعد على زيادة التفاعل الكيميائي مما يمنع تكون التجمعات الكبيرة (الرواسب) على سطح النموذج وبالتالي تقلل من سمك الغشاء، بينما لوحظ بأن سمك الغشاء يزداد مـع زيادة زمن الترسيب

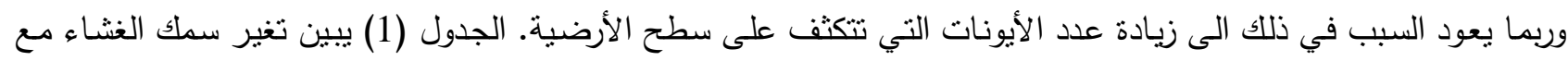
ظروف التحضير أعلاه.

الجدول 1: علاقة سمك الغشاء بتغير زمن الترسيب ودرجة الحرارة

\begin{tabular}{|c|c|c|c|c|}
\hline \multirow{4}{*}{$\mathrm{ZnS}$} & زمن الترسيب & سمك الفثاء & 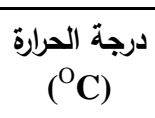 & سمك الغثاء \\
\hline & 30 & 100 & 40 & 197 \\
\hline & 60 & 247 & 55 & 148 \\
\hline & 90 & 494 & 70 & 100 \\
\hline
\end{tabular}

الخواص التركيبية

حيود الأشعة السينية

تبين الأشكال (1 و2) نتائج حيود الأشعة السينية (XRD) لغشاء ZnS النانوي المحضر بطريقة الحمام الكيميائي (CBD) حيث يظهر من طيف الأثعة السينية بان الغشاء ذو تركيب عشوائي (Amorphous) ولجميع ظروف التحضير مع أختلاف (Aamodt, 2011 ; Salleh et al., 2010; Abdullah et al., 2012; قليل جداً في الثدة وهذا يتفق مع نتائج الباحثين لئنين وربما يرجع السبب في ذلك لكون الترسيب بهذه الطريقة ينت عند درجات حرارة منخفضة قريبة من درجة 


$$
\text { تحسين علي أسود و آخرون }
$$

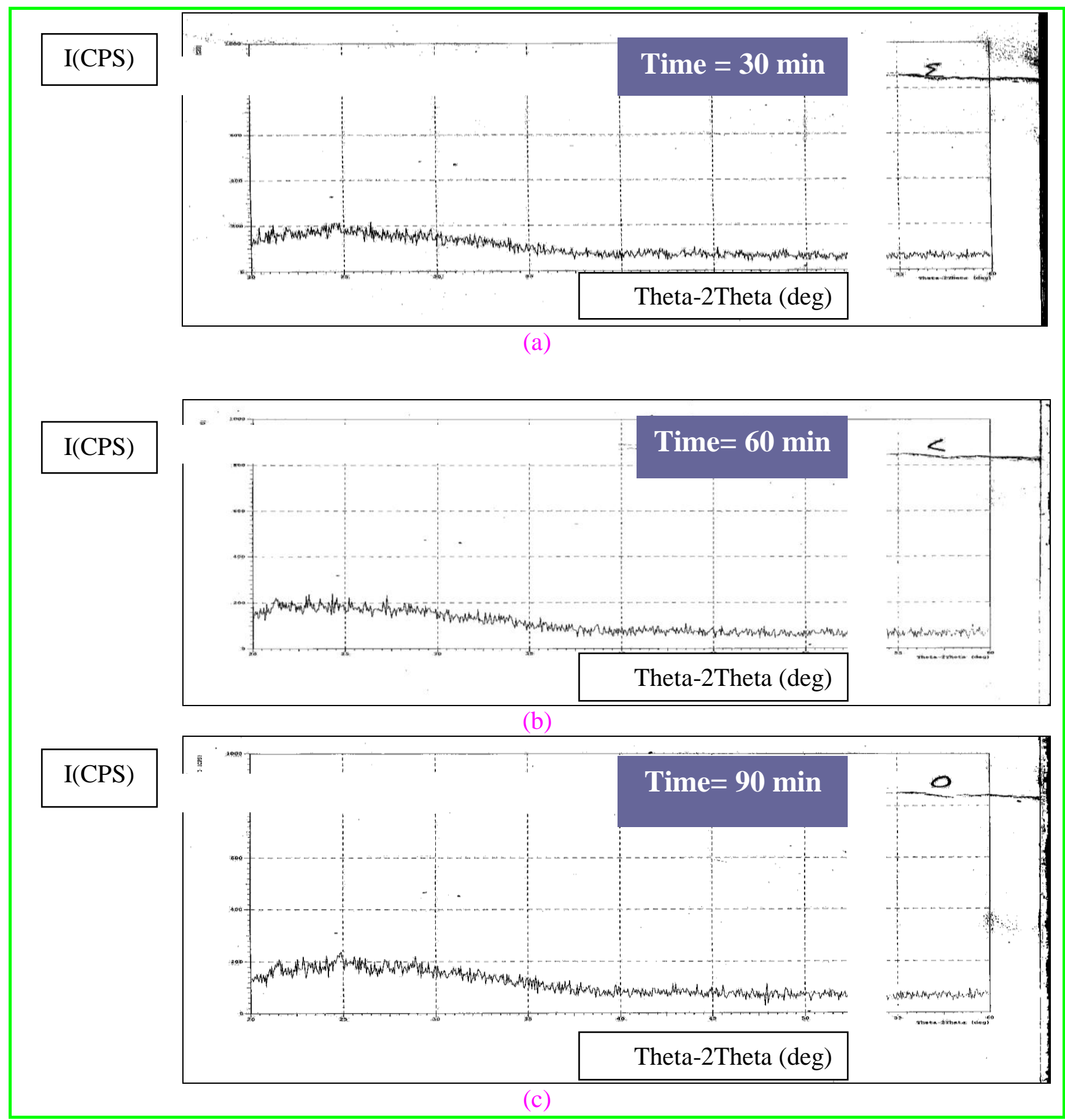

الثكل 1: حيود الأثعة السينية لغثاء ZnS النانوي المحضر بطريقة (CBD) (CBD) لأزمان مختلفة:

90 min (c) $\quad 60$ min (b) $\quad 30$ min (a)




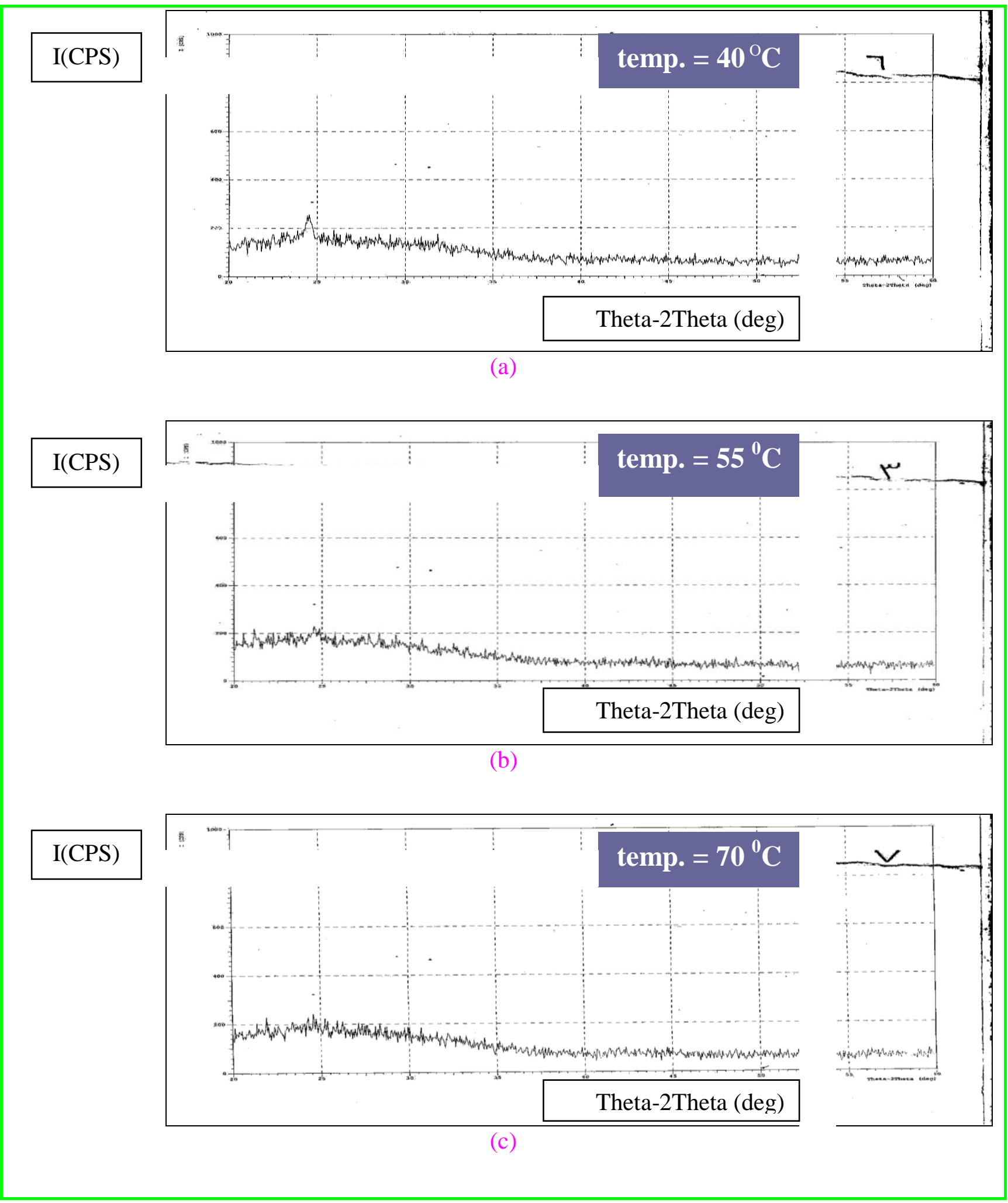

الثكل 2: حيود الأثعة السينية لغشاء ZnS النانوي المحضر بطريقة (CBD) للارجات حرارية مختلفة:

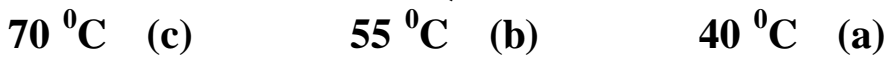


الخواص الطبوغرافية

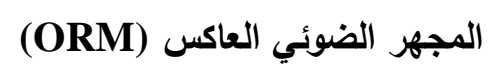

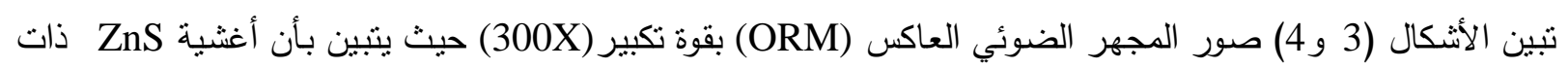

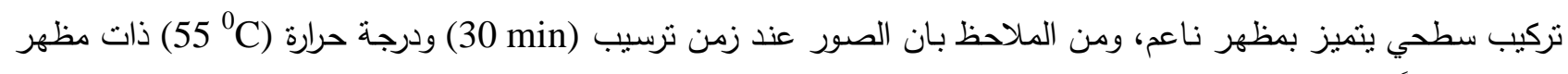

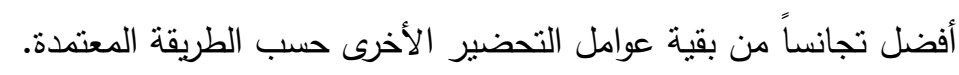

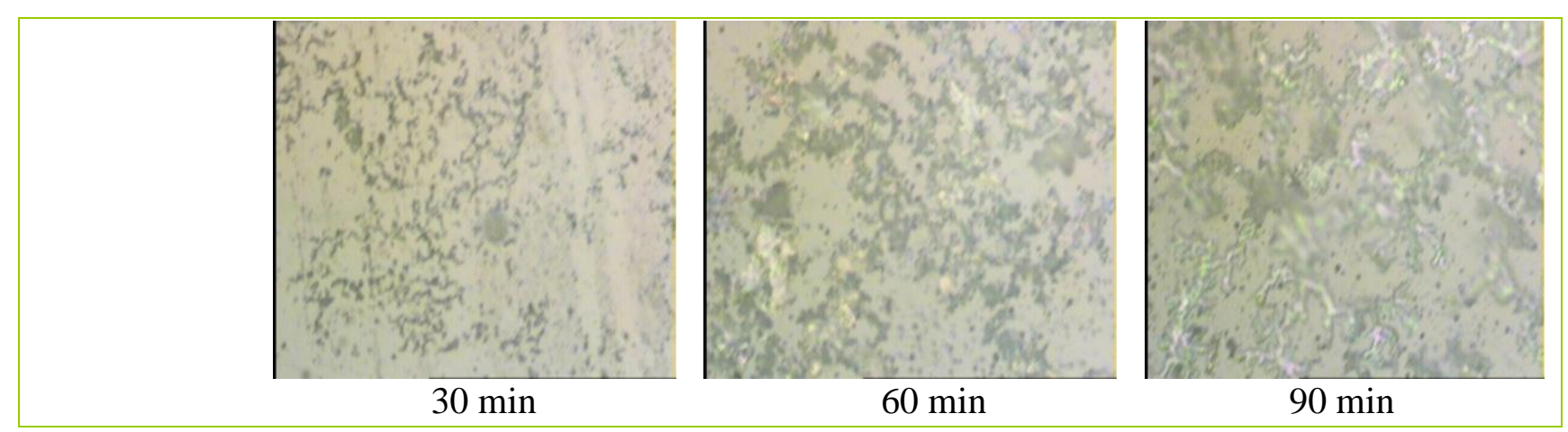

الثكل 3: صور المجهر الضوئي (ORM) عثد أزمان ترسيب مختلفة

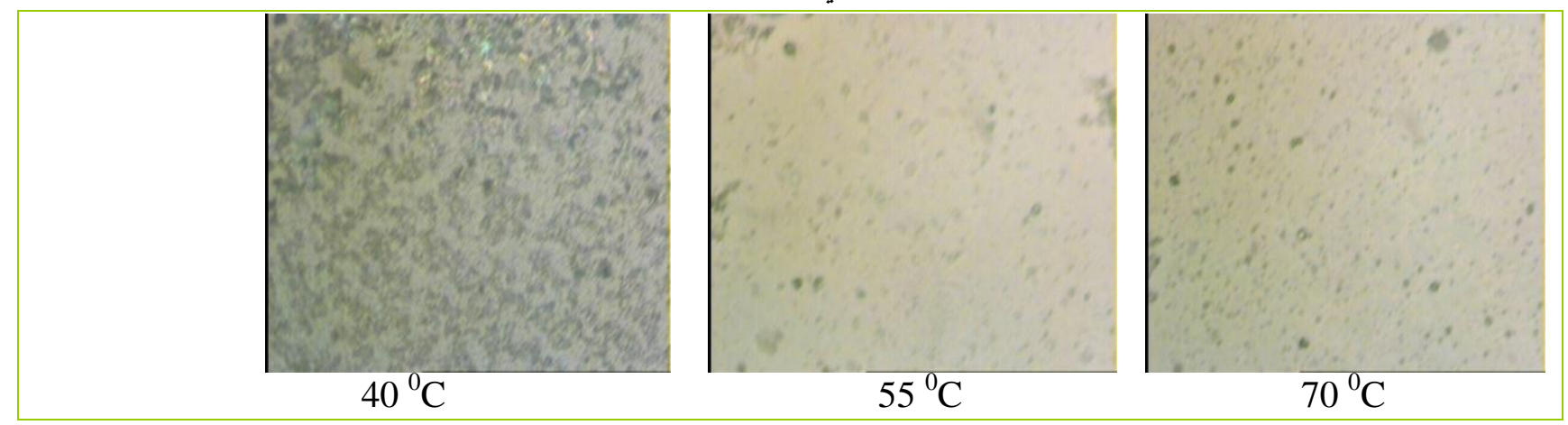

الثكل 4: صور المجهر الضوئي (ORM) عند درجات حرارية مختلفة

مجهر القوة الذرية (AFM)

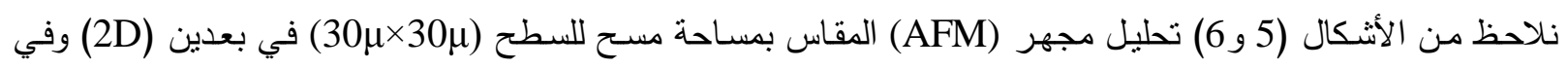

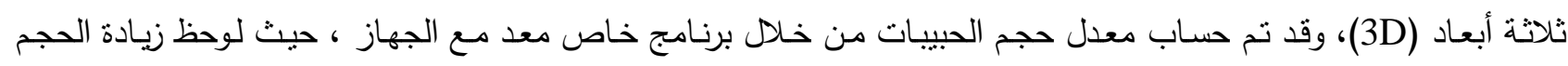

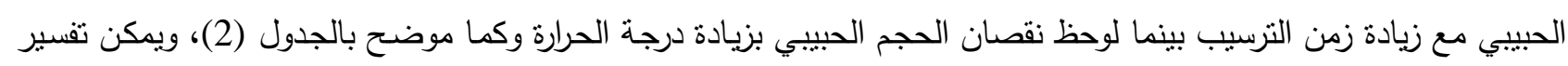

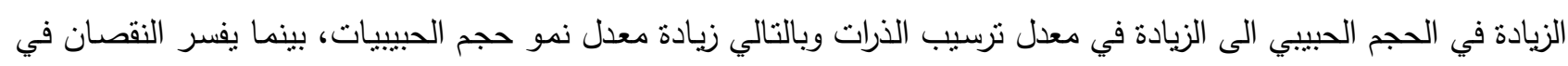
الحجم الحبيبي على العكس من ذللك.

الجدول 2: علاقة الحجم الحبيبي بتغير زمن الترسيب ودرجة الحرارة

\begin{tabular}{|c|c|c|c|c|}
\hline \multirow{4}{*}{$\mathrm{ZnS}$} & 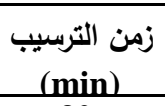 & $\begin{array}{c}\text { الحجم الحبييبي } \\
\text { (nm) }\end{array}$ & 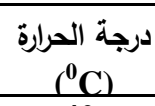 & $\begin{array}{c}\text { الحجم الحبيبي } \\
\text { (nm) }\end{array}$ \\
\hline & & 63.3 & 40 & 66.8 \\
\hline & 60 & 66 & 55 & 65 \\
\hline & 90 & 77.6 & 70 & 63.9 \\
\hline
\end{tabular}


تأثثر زمن التزسيب ودرجة الحرارة على الخصائص

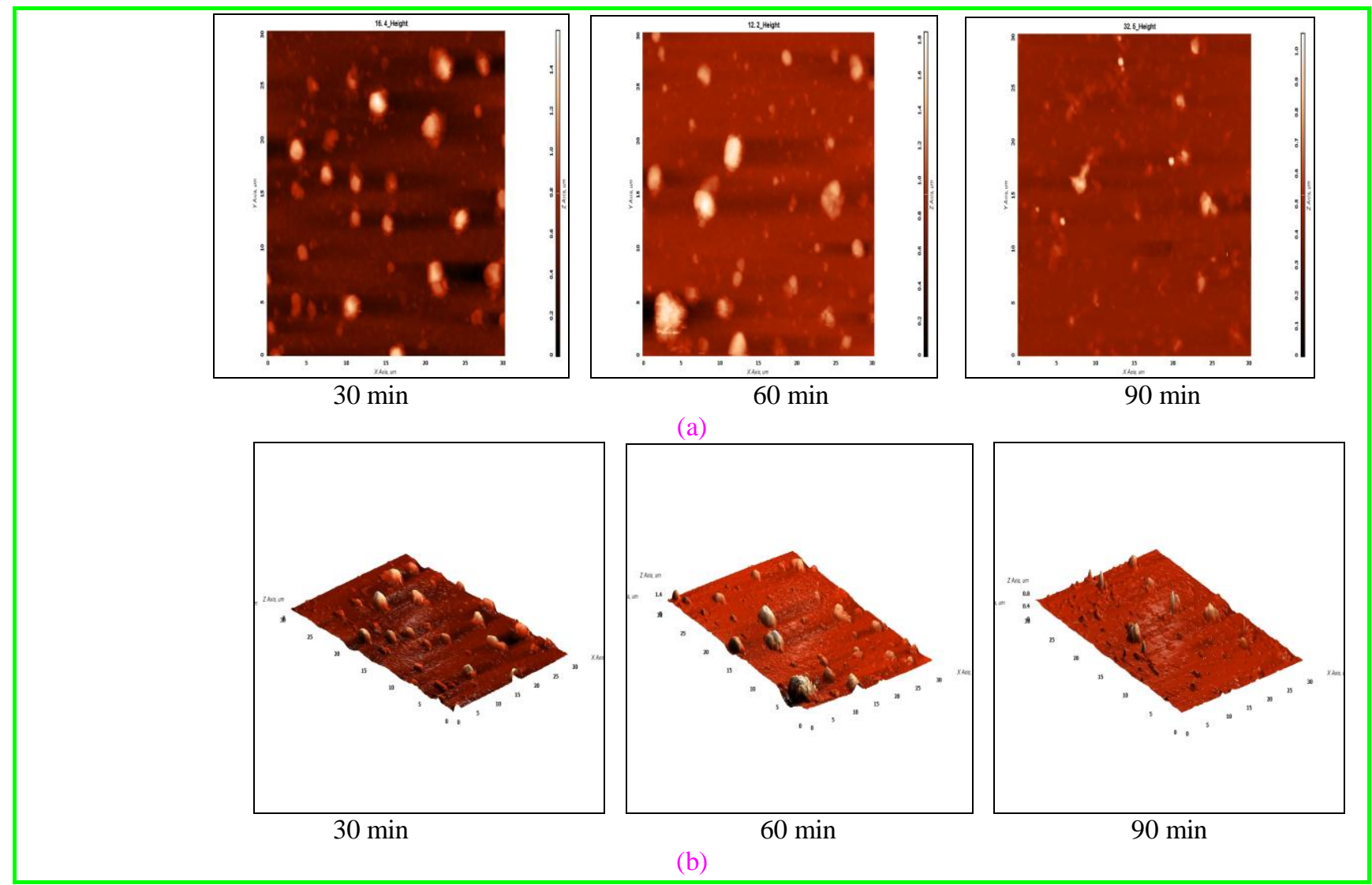

الثكل 5: صور مجهر القوة الذرية عند أزمان ترسيب مختلفة: (a) ببعدين (2-D) (b) بثلاثة أبعاد (3-D) (b)
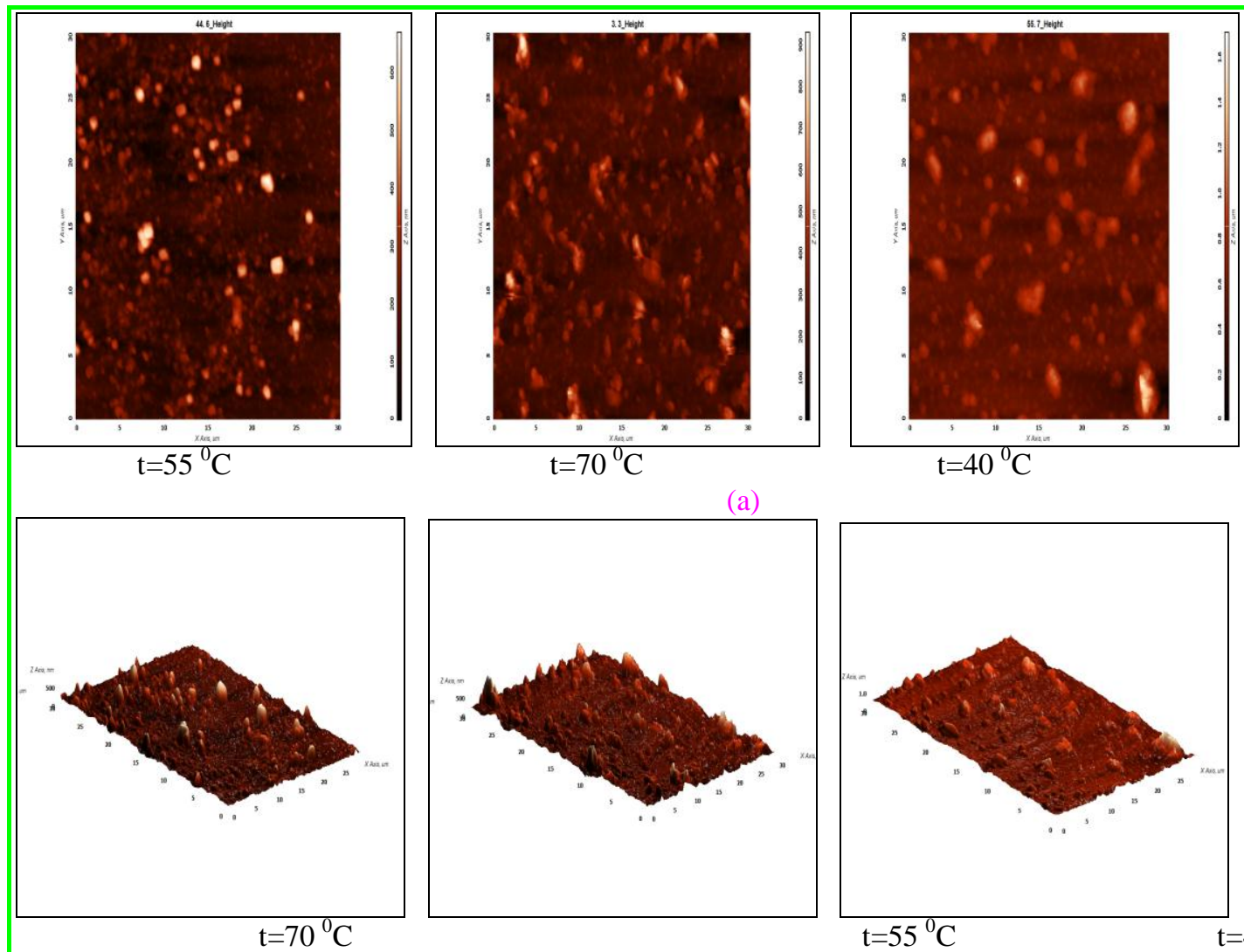

(a)
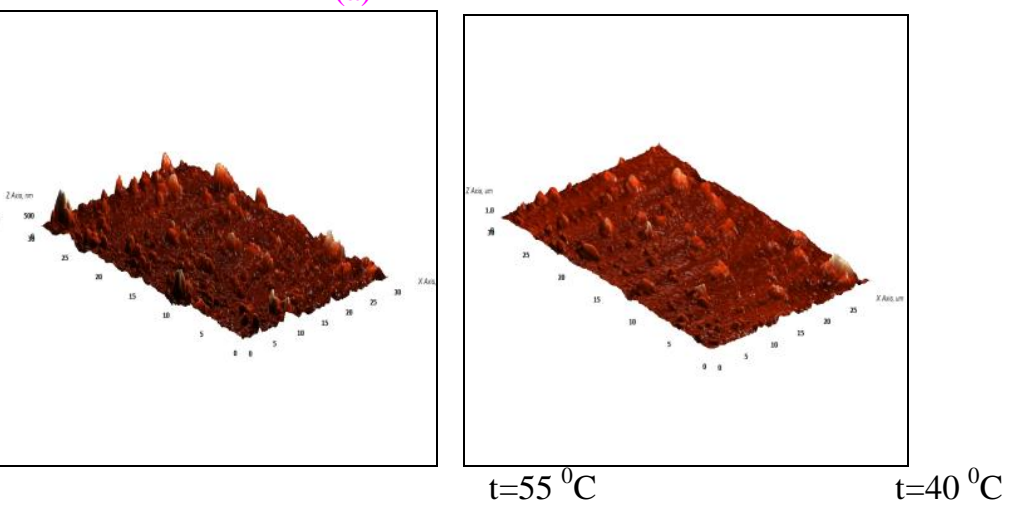

(b)

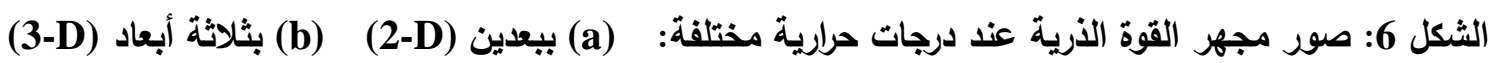




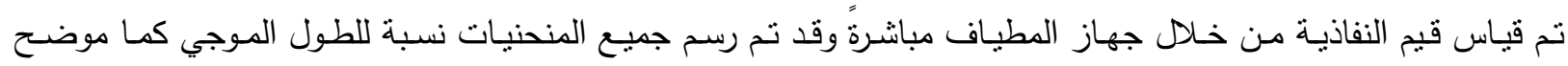

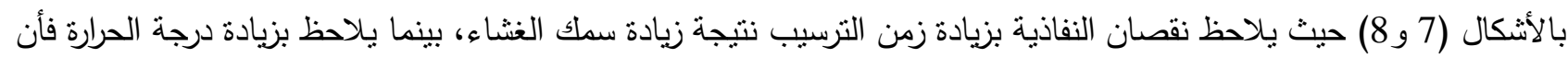
قيمة النفاذية تزداد ثم تتناقص وهذا ريما يعود الى التأثير الكبير للحرارة على التفاعل الكيميائي.

$\mathrm{T}$

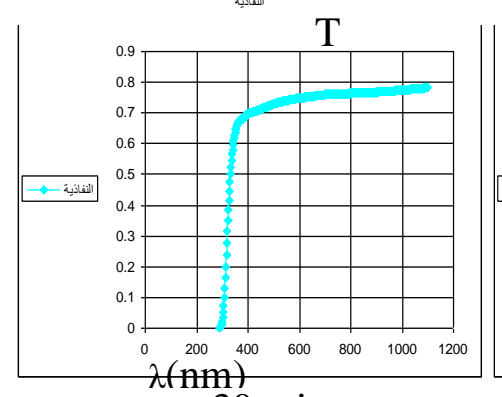

$30 \min$

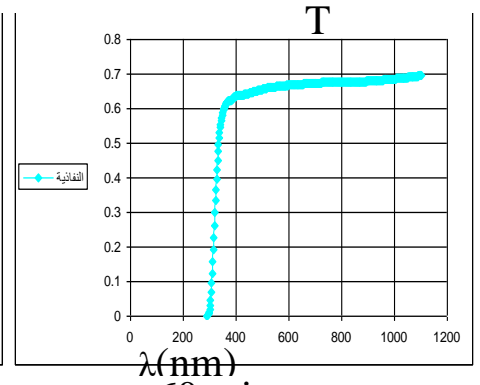

$60 \mathrm{~min}$

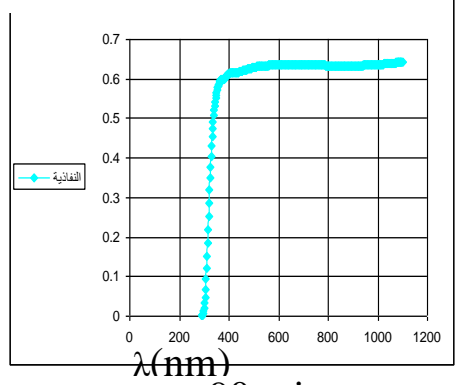

$90 \min$

الثكل 7: قيم النفاذية كدالة للطول الموجي عند أزمان ترسيب مختلفة

$\mathrm{T}$

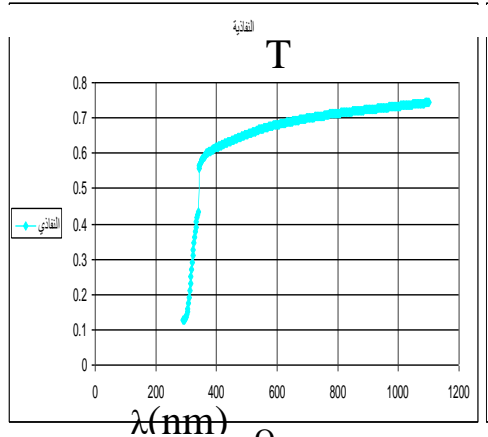

$40{ }^{\circ} \mathrm{C}$

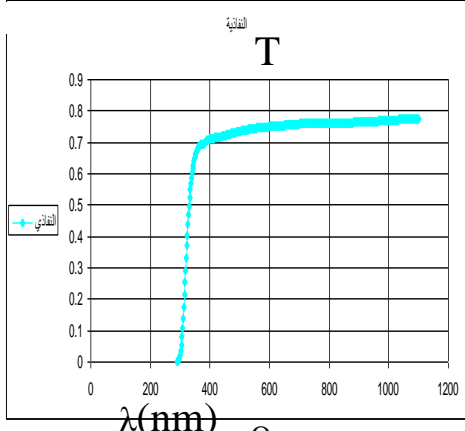

$55^{\circ} \mathrm{C}$

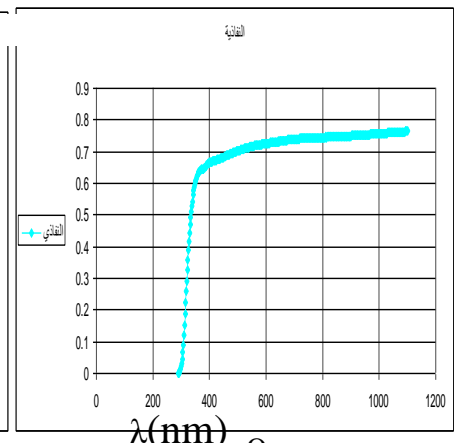

$70{ }^{\circ} \mathrm{C}$

الثكل 8: قيم النفاذية كدالة للطول الموجي عند درجات حرارية مختلفة

فجوة الطاقة (Eg):

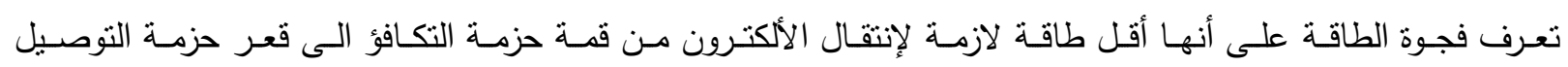

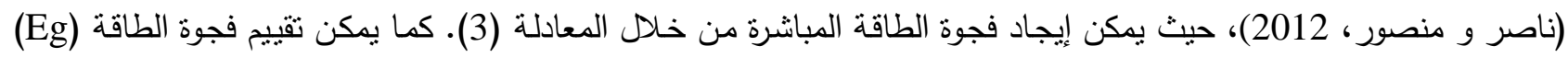

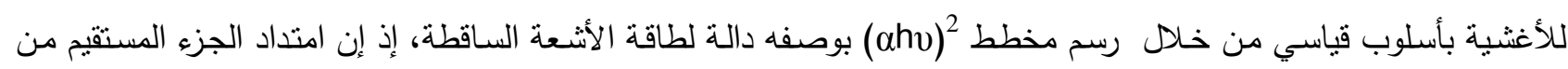

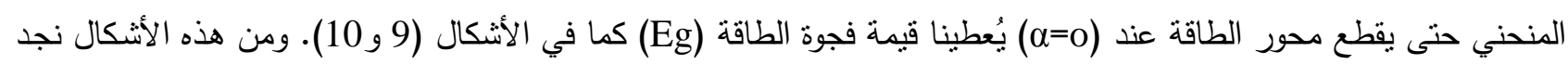

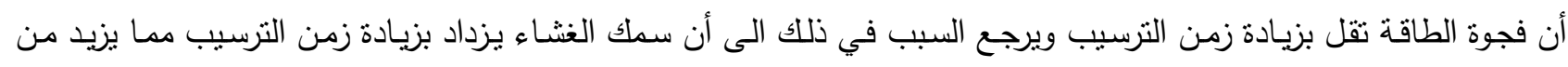

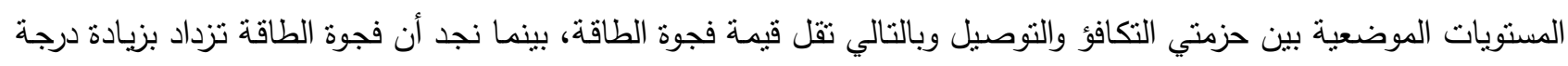

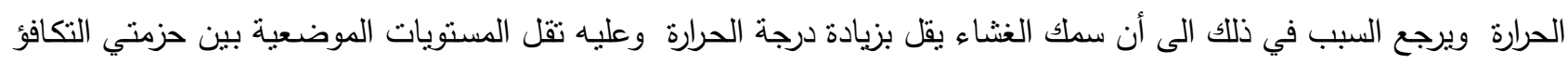
والتوصيل وبالنالي تزداد قيمة فجوة الطاقة. والجدول (3) يوضح قيم فجوة الطاقة ولجميع الحالات. 
الجدول 3: علاقة فجوة الطاقة بتغير زمن الترسيب ودرجة الحرارة

\begin{tabular}{|c|c|c|c|c|}
\hline \multirow{4}{*}{$\begin{array}{c}\mathrm{ZnS} \\
\text { Nano Film }\end{array}$} & $\begin{array}{l}\text { زمن الترسيبب (min) } \\
\text { (min }\end{array}$ & $\begin{array}{c}\text { فوة الطاقة } \\
\text { (eV) }\end{array}$ & $\begin{array}{c}\text { درجة الحرارة } \\
\text { ('C) }\end{array}$ & فجوة الطاقة \\
\hline & 30 & 3.87 & 40 & 3.2 \\
\hline & 60 & 3.83 & 55 & 3.9 \\
\hline & 90 & 3.8 & 70 & 3.95 \\
\hline
\end{tabular}

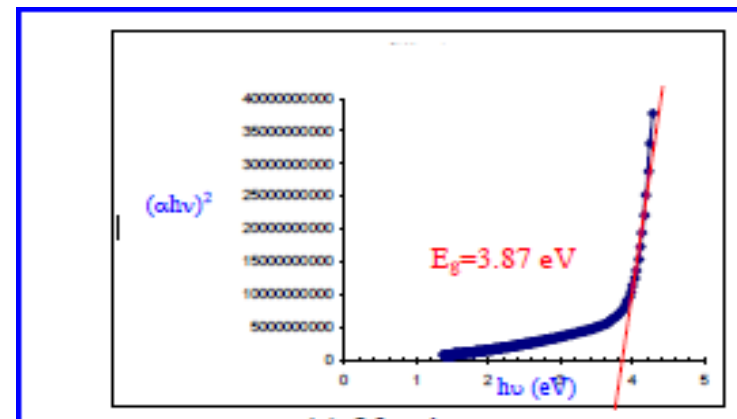

(a) $30 \mathrm{~min}$

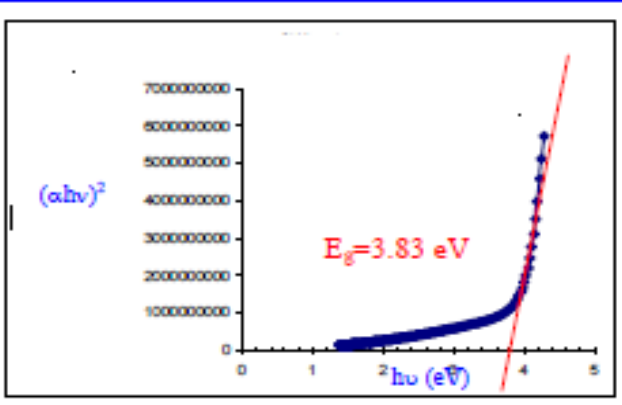

(b) $60 \mathrm{~min}$

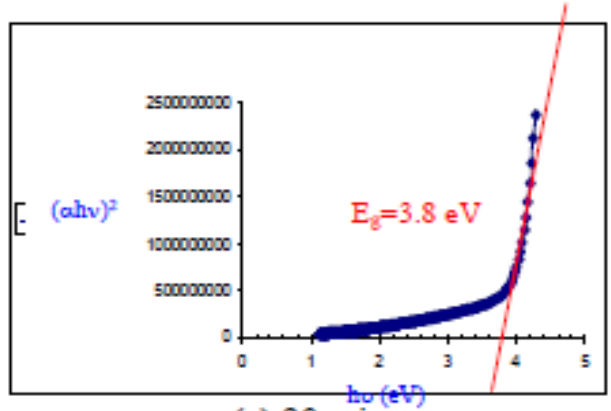

(c) $90 \mathrm{~min}$

الشكل 9: التغير في قيم

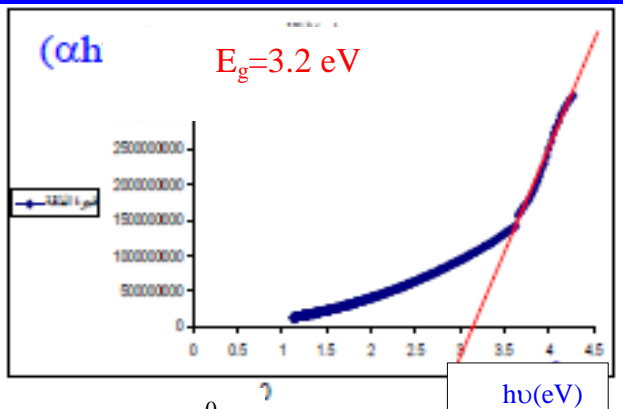

(a) $40{ }^{\circ} \mathrm{C}$

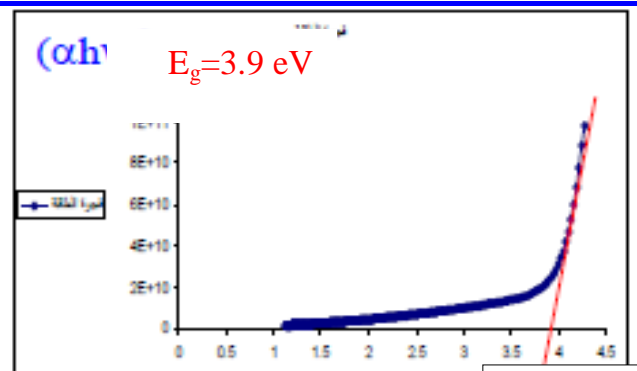

(b) $55{ }^{\circ} \mathrm{C}$

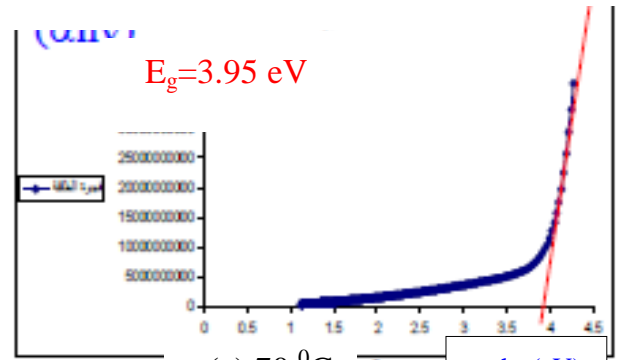

(c) $70{ }^{\circ} \mathrm{C}=\mathrm{hu}(\mathrm{eV})$

الثكل 10: التغير في قيم 
معامل الامتصاص

تم حساب قيم معامل الامتصاص بأستخدام المعادلة (1) وقد تم رسم جميع المنحنيات نسبة للطول الموجي ومن ملاحظة

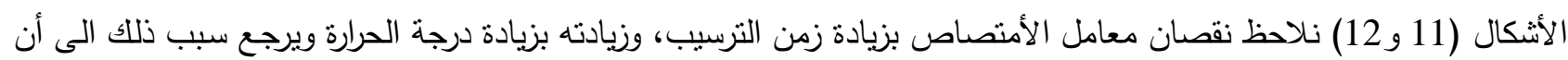

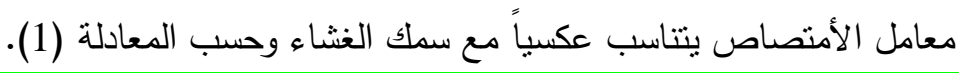

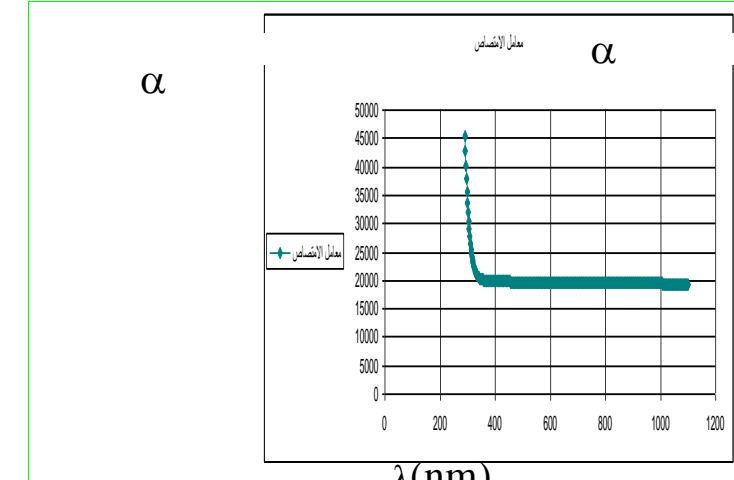

$\lambda(\mathrm{nm})$

$30 \mathrm{~min}$

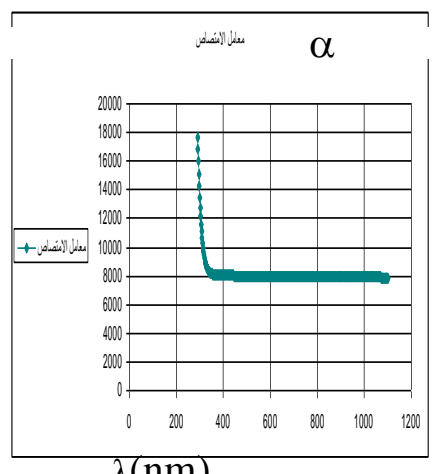

$\lambda(\mathrm{nm})$

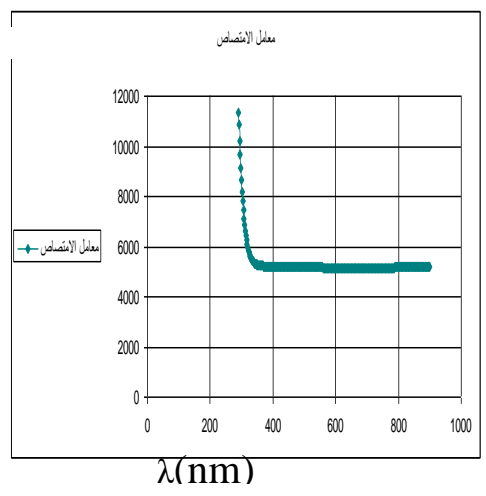

$90 \mathrm{~min}$

الشكل 11: قيم معامل الأمتصاص كدالة للطول الموجي عند أزمان ترسيب مختلفة

$\alpha$

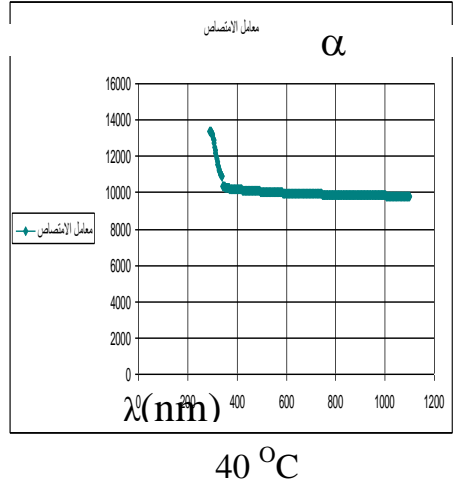

$60 \mathrm{~min}$

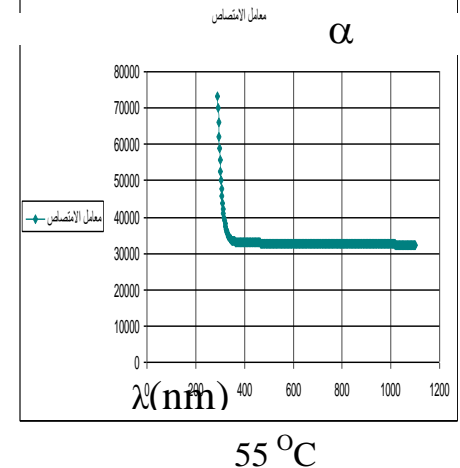

$55{ }^{\circ} \mathrm{C}$

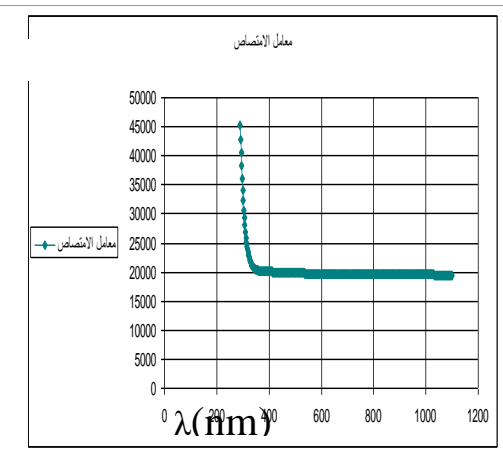

$70{ }^{\circ} \mathrm{C}$

الشكل 12: قيم معامل الأمتصاص كدالة للطول الموجي عند درجات حرارية مختلفة

معامل الخمود

تم حساب قيم معامل الخمود بأستخدام المعادلة (2) وقد تم رسم جميع المنحنيات نسبة للطول الموجي ومن ملاحظة

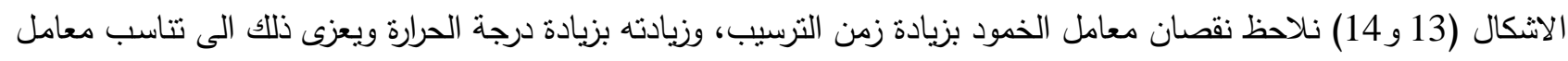

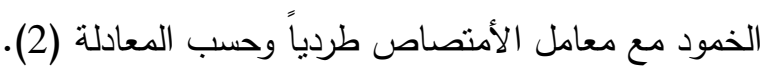

$\mathrm{k}_{0}$

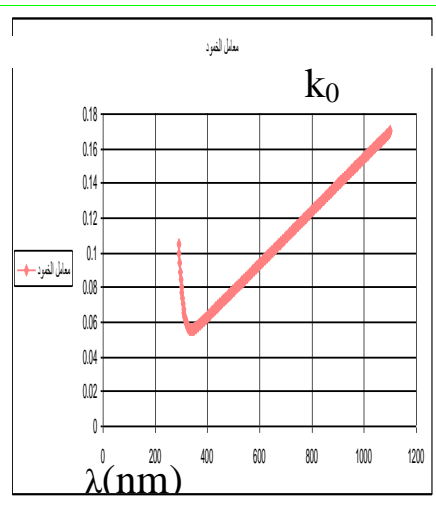

$30 \mathrm{~min}$

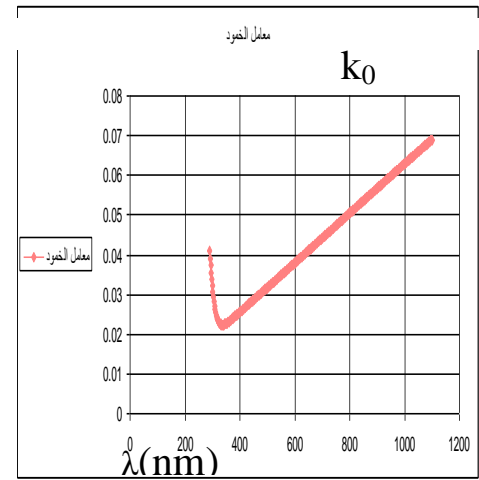

60 min

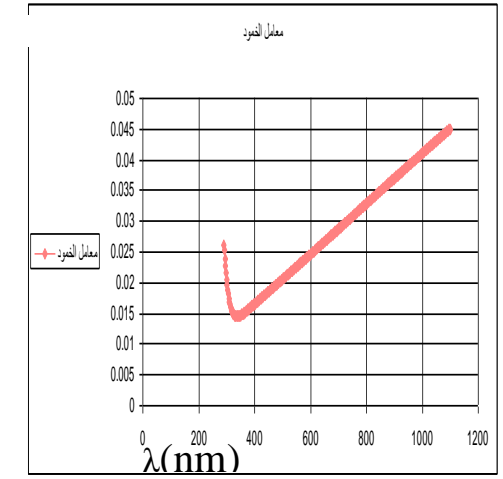

$90 \mathrm{~min}$

الشكل 13: قيم معامل الخمود كدالة للطول الموجي عند أزمان ترسيب مختلفة 
$\mathrm{k}_{0}$
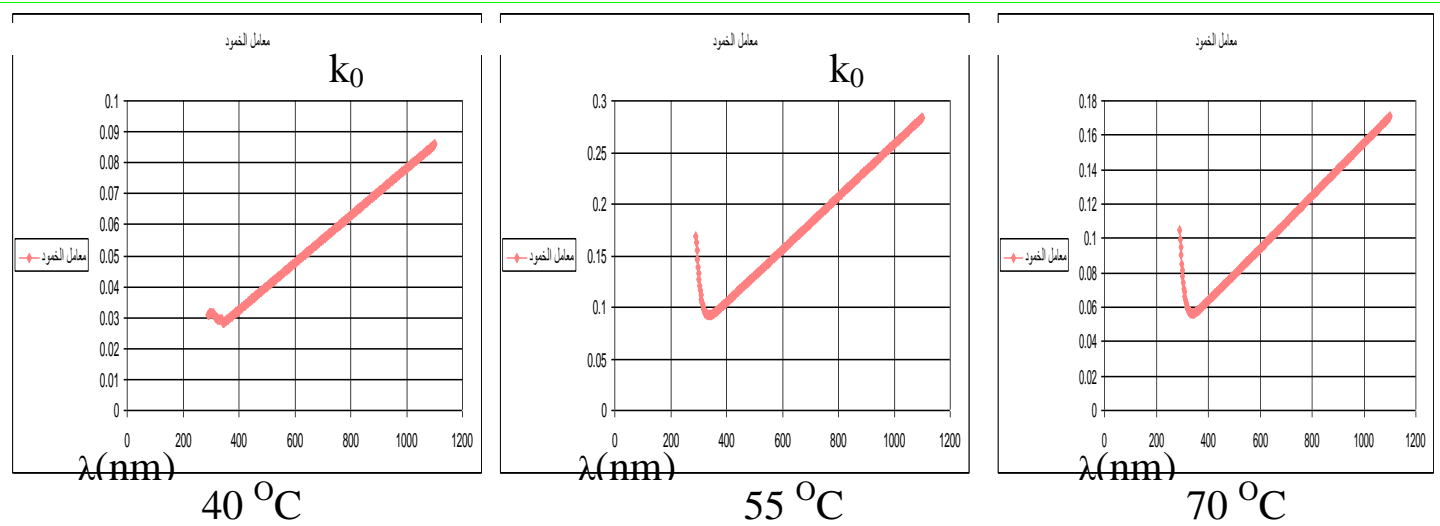

الثكل 14: قيم معامل الخمود كدالة للطول الموجي عند درجات حرارية مختلفة

الاستنتاجات

أدى زيادة زمن الترسيب إلى تقليل قيمة فجوة الطاقة الممنوعة للانتقال المباشر المسموح بينما أدت زيادة درجة الحرارة إلى زيادة قيمة فجوة الطاقة الممنوعة للانتقال المباشر المسموح، كما لوحظ أن زمن الترسيب ودرجة الحرارة لهما تأثير واضح على بقية الثوابت البصرية (النفاذية، معامل الأمنصاص، معامل الخمود) أذ نقل هذه الثثابت بزيادة زمن الترسيب وتزداد بزيادة درجة الحرارة. المصادر العربية

الجمال، يحيى نوري؛ ال فليح، رنا زياد (2006). الخواص الكهبائية للمنسعات ذات الغشاء الرقيق من السبائك البوليميرية، مجلة علوم الرافدين، 17(2)، 33- 44.

ناصـر ، باسـل هاثــ؛ منصسور ، ميخائيل عيسى (2012). دراسـة الخصـائص الفيزيائيـة لأغثـية أوكسيد الخارصـين المطعهـة

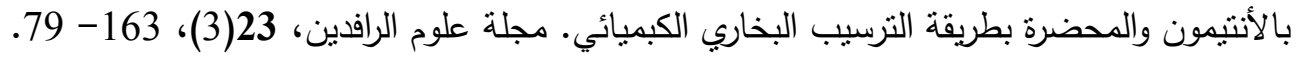

\section{المصادر الأجنبية}

Abdullah, H.; Saadah, N.; Shaari, S. (2012). "Effect of Deposition Time on ZnS Thin Films Properties by Chemical Bath Deposition (CBD) Technique". Malaysia. 1088 p.

Chandran, A.; Francis, N.; Jose, T.; George, K.C. (2010). Synthesis, structural characterization and optical band gap determination of ZnS Nano- particles. Changanacherry, XVII(1), 17-21.

Divya, A.; Reddy, B.K.; Sambasivam, S.; Sreedhara, P. (2011). Photolumin- essence and EPR studies of ZnS Nanoparticles Co-Doped with Mn and Te. India , 3(1), 639.

Eya, D.O.; Ekpunobi, A.J.; Okeke, C.E. (2005). Structural and optical properties and applications of Zinc Oxide thin films prepared by chemical bath deposition technique. Pac. J. Sci. \& Tech. , 6 (1).

Fang, X.; Zhai, T.; Gautam, U.K.; Li, L.; Wu, L.; Bando, Y.; Golberg, D. (2011). ZnS nanostructures: From synthesis to applications. China, 56,177.

Gayou1, V.L.; Salazar-Hernández, B.; Delgado Macuil, R.; Zavala, G.; Santiago, P.; Oliva, A.I. (2010). Structural studies of $\mathrm{ZnS}$ nano particles by high resolution transmission electron microscopy. J. Nano Research. 9, 125- 132, México.

Guixiang, Q. (2009). "Controlled Fabrication, Characterization and Properties of One-dimensional Semiconductor Nanostructures from and on Metal Substrates". Hong Kong

Li, L.S.; Alivisatos, A.P. (2003). Semiconductor nanorod liquid crystals and their assembly on a substrate. Adv. Mater., 15, 408.

Ma, C.; Moore, D.; Ding, Y.; Li, J.; Wang, Z.L. (2004). Nanobelt and nanosaw structures of II-VI semiconductors. Int. J. Nanotechnology, 1(4). 
Nadeem, M.Y.; Ahmed, W.; Wasiq, M.F. (2005). ZnS Thin Films - an overview. Pakistan,16(2), 105-112.

Salleh, S.; Dalimin, M.N.; Rutt, H.N. (2010). "Structural and Surface Characterization of Cold Deposited Zinc Sulfide Thin Films". $3^{\text {rd }}$ International Conference on Solid State Science and Technology, Malaysia.

Stenzel, O. (2005). "The Physics of Thin Film Optical Spectra". An Introduction, Winzerlaer Str. 10 , Germany .

Suresh, S. (2013). Synthesis structural and dielectric properties of zinc sulfide nanoparticles. India, 8(21), 1121.

Thottoli, A.; Unni, A.K.A. (2013). Effect of polyvinyl alcohol concentration on the $\mathrm{ZnS}$ nanoparticles and wet chemical synthesis of wurtzite $\mathrm{ZnS}$ nanoparticles. India .

Tor Ingve Aamodt (2011). "Characterization of ZnS: Cr Films for Intermediate Band Solar Cells". Teacher Education with Master of Science, Norwegian University of Science and Technology.

Üzar, N.; Arikan, M. (2010). Synthesis and investigation of optical properties of ZnS nanostructures. Turkey, 34(2), 287.

Wang, J.; Li, Y. (2003). Rational synthesis of metal nanotubes and nanowires from lamellar structures. Adv. Mater., 15, 445.

Xu, C.; Xue, L.; Yin, C.; Wang, G. (2003). Formation and photoluminescence properties of AlN nanowires. Phys. Stat. Sol. A., 198, 329.

Yu-Bin, Chen. (2012). Introduction to nanotechnology-history, definition, methodology, applications, and challenges". Nonsocial Engineering Radiation Lab, 10 p. 